\title{
CYP2D6 phenotypes are associated with adverse outcomes related to opioid medications
}

This article was published in the following Dove Press journal:

Pharmacogenomics and Personalized Medicine

24 July 2017

Number of times this article has been viewed

\author{
Jennifer L St Sauver ${ }^{1,2}$ \\ Janet E Olson ${ }^{1,3}$ \\ Veronique L Roger ${ }^{1,2,4}$ \\ Wayne T Nicholson ${ }^{5}$ \\ John L Black III ${ }^{3,6}$ \\ Paul Y Takahashi ${ }^{7}$ \\ Pedro J Caraballo 7 \\ Elizabeth J Bell ${ }^{2}$ \\ Debra J Jacobson ${ }^{1,2}$ \\ Nicholas B Larson' \\ Suzette J Bielinski ${ }^{1,3}$ \\ 'Department of Health Sciences \\ Research, ${ }^{2}$ Kern Center for the \\ Science of Health Care Delivery, \\ Mayo Clinic, Rochester, ${ }^{3}$ Center for \\ Individualized Medicine, ${ }^{4}$ Department \\ of Cardiovascular Diseases, \\ ${ }^{5}$ Department of Anesthesiology and \\ Perioperative Medicine, ${ }^{6}$ Department \\ of Laboratory Medicine and Pathology, \\ ${ }^{7}$ Department of Primary Care Internal \\ Medicine, Mayo Clinic, Rochester, \\ MN, USA
}

Correspondence: Jennifer L St Sauver Department of Health Sciences Research, Mayo Clinic, 200 First Street SW, Rochester, MN 55905, USA

$\mathrm{Tel}+\mathrm{I} 507538$ I460

Fax + I 507284 I5I6

Email stsauver.jennifer@mayo.edu
Background: Variation in the CYP2D6 gene may affect response to opioids in both poor and ultrarapid metabolizers, but data demonstrating such associations have been mixed, and the impact of variants on toxicity-related symptoms (e.g., nausea) is unclear. Therefore, we examined the association between $C Y P 2 D 6$ phenotype and poor pain control or other adverse symptoms related to the use of opioids in a sample of primary care patients.

Materials and methods: We identified all patients in the Mayo Clinic RIGHT Protocol who were prescribed an opioid medication between July 01, 2013 and June 30, 2015, and categorized patients into three phenotypes: poor, intermediate to extensive, or ultrarapid CYP2D6 metabolizers. We reviewed the electronic health record of these patients for indications of poor pain control or adverse symptoms related to medication use. Associations between phenotype and outcomes were assessed using Chi-square tests and logistic regression.

Results: Overall, 257 (25\% of RIGHT Protocol participants) patients received at least one opioid prescription; of these, 40 (15\%) were poor metabolizers, $146(57 \%)$ were intermediate to extensive metabolizers, and $71(28 \%)$ were ultrarapid metabolizers. We removed patients that were prescribed a CYP2D6 inhibitor medication $(n=38)$. After adjusting for age and sex, patients with a poor or ultrarapid phenotype were 2.7 times more likely to experience either poor pain control or an adverse symptom related to the prescription compared to patients with an intermediate to extensive phenotype (odds ratio: $2.68 ; 95 \% \mathrm{CI}: 1.39,5.17 ; p=0.003$ ).

Conclusion: Our results suggest that $>30 \%$ of patients with a poor or ultrarapid CYP2D6 phenotype may experience an adverse outcome after being prescribed codeine, tramadol, oxycodone, or hydrocodone. These medications are frequently prescribed for pain relief, and $\sim 39 \%$ of the US population is expected to carry one of these phenotypes, suggesting that the population-level impact of these gene-drug interactions could be substantial.

Keywords: CYP2D6, opioid, phenotype, adverse effects

\section{Plain language summary}

Prescription of opioid pain medications in the USA is very high; between 2011 and 2012, nearly $7 \%$ of the adult population was estimated to have taken an opioid in the last 30 days. However, these medications do not control pain in all patients, and some patients are likely to have problems such as vomiting or breathing difficulties after taking an opioid. Characterizing the factors that predict individual patient response to opioid analgesics could help to better target these medications to the patients mostly likely to benefit, while reducing side effects in patients most likely to have problems. The field of pharmacogenomics offers the opportunity to identify patients who carry genetic variants that are likely to impact their response to these medications. Therefore, we studied the impact of variation in the CYP2D6 gene on patient 
responses to codeine, tramadol, oxycodone, or hydrocodone. Our results suggest that $>30 \%$ of patients with a poor or ultrarapid CYP2D6 phenotype may experience an adverse outcome after being prescribed one of these medications. Nearly $40 \%$ of the US population is expected to carry one of these phenotypes, suggesting that a large number of people could benefit from more targeted opioid prescribing.

\section{Introduction}

Opioid analgesics are effective, commonly prescribed medications used for the management of both acute and chronic pain in patients with many different medical conditions and following many medical procedures. ${ }^{1,2}$ Prescription of opioids in the USA is high, and between 2011 and 2012, nearly 7\% of the adult population was estimated to have taken an opioid in the last 30 days. ${ }^{3,4}$ Opioid use is especially prevalent among older adults; in 2010, an estimated 9\% of adults over 65 years took opioid medications. ${ }^{5}$ However, these medications do not effectively control pain in all patients. ${ }^{6-10}$ In addition, many patients are at high risk of adverse effects due to these medications. ${ }^{8,10}$ A meta-analysis of randomized trials found that $80 \%$ of patients treated with opioids for chronic, noncancer pain experienced at least one adverse event, with symptoms ranging from mild nausea to life-threatening respiratory depression. ${ }^{8}$

Characterizing the factors that predict individual patient response to opioid analgesics could help to better target prescriptions to the patients mostly likely to benefit from these medications. The field of pharmacogenomics offers the opportunity to identify specific individual characteristics that could predict such responses. ${ }^{11,12}$ In particular, the cytochrome P450 2D6 (CYP2D6) enzyme is involved in the prodrug conversion of codeine, oxycodone, hydrocodone, and tramadol. ${ }^{13,14}$ Patients with an ultrarapid CYP2D6 phenotype are likely to experience higher systemic levels of the active metabolites of these analgesics upon treatment, while patients with a poor metabolizer $C Y P 2 D 6$ phenotype will tend to have lower levels. ${ }^{13,15}$ Therefore, a patient's CYP2D6 phenotype may result in reduced efficacy in poor metabolizers. ${ }^{16}$

Conversely, there is some evidence that ultrarapid metabolizers require less pain medication to achieve pain control. ${ }^{17}$ These patients may, however, be at a greater risk of respiratory depression at standard doses compared to normal metabolizers. ${ }^{12,18}$ However, data demonstrating associations between $C Y P 2 D 6$ phenotype and poor pain control have been mixed. ${ }^{13,16}$ Several studies have indicated that persons with a poor metabolizer phenotype experience less pain relief from codeine and tramadol, ${ }^{19-25}$ but the effect of a poor metabolizer phenotype on pain relief from oxycodone and hydrocodone is less clear. ${ }^{26-30}$ In addition, the impact of CYP2D6 variants on mild, nonlife threatening, adverse reactions associated with opioids (e.g., nausea), and subsequent effects on pain control due to intolerance, has not been routinely studied. ${ }^{16}$ A larger study of cancer patients found no differences in pain control, nausea, or tiredness among poor, normal, and ultrarapid metabolizers taking oxycodone. ${ }^{30}$ It is not clear, however, whether these results in cancer patients are generalizable to all patients prescribed these medications. Finally, previous studies have focused on a single medication at a time, and it is not clear whether the results of these studies represent the experience of a day-to-day clinical population.

Data regarding the impact of CYP2D6 phenotypic variation on a general population in real-world clinical practice are limited. To address this gap, we examined the association between CYP2D6 phenotype and poor pain control or other adverse symptoms related to the use of opioid analgesics among participants in the Mayo Clinic Right Drug, Right Dose, Right Time Protocol (RIGHT Protocol) study. ${ }^{31}$ Participants in this study are patients enrolled in the Mayo Clinic general internal medicine practice and could receive opioid prescriptions for a wide range of indications. We used this population to study the association between CYP2D6 phenotype and opioid prescription outcomes in a real-world clinical setting.

\section{Materials and methods Setting and study participants}

Details of the RIGHT Protocol study have been previously reported. ${ }^{31}$ Briefly, 2,000 participants in the Mayo Clinic Biobank were invited to participate in a study of pre-emptive genotyping; 1,013 (51\%) agreed to participate. ${ }^{32}$ Participants contributed a blood sample that was used to sequence genetic variants in the CYP2D6 gene that could cause drug-gene interactions with opioid analgesics. At the time of enrollment, participants in the RIGHT study provided written informed consent to allow their PGx genetic data and electronic health record data to be used for research in future PGx studies. This study was approved by the Mayo Clinic and Olmsted Medical Center Institutional Review Boards.

We used the Rochester Epidemiology Project research infrastructure to identify all patients in the RIGHT Protocol who received an opioid analgesic prescription between $07 / 01 / 2013$ and 06/30/2015 from a local health care provider. ${ }^{33,34}$ Specifically, we identified all RxNorm codes 
for prescriptions that included the ingredients codeine, oxycodone, hydrocodone, and tramadol, because the metabolic activation of these medications can be affected by CYP2D6 variation. ${ }^{13,14}$ We excluded formulations intended primarily to treat cough (e.g., guaifenesin/codeine). A complete list of medications considered is shown in Table S1. In addition, we identified all other prescriptions received on the same day. Approximately $25 \%$ of the population received one or more prescriptions in addition to the opioid, and the types of other prescriptions are shown in Table $\mathrm{S} 2$.

\section{CYP2D6 genotyping}

CYP2D6 was genotyped using a custom-designed testing cascade, beginning with the Luminex Tag-It Mutation Detection Kit for Cytochrome P450 2D6 (CYP2D6 ASPE Kit v2; Luminex Corporation, Austin, TX, USA) and, when needed, followed by a laboratory-developed copy number variation assay and/or sequencing assays. ${ }^{35}$

\section{CYP2D6 phenotyping}

Methods for interpreting pharmacogenomic variants have been previously described. ${ }^{36}$ Phenotype prediction was binned into four categories in a manner similar to that reviewed by Ingelman-Sundberg and Kirchheiner et al. ${ }^{37,38}$ It should be noted that other methodologies for phenotype prediction have been described, and this is a controversial area of pharmacogenomics. ${ }^{39,40}$ In particular, there is controversy about where to classify the $C Y P 2 D 6^{*} 2 A$ versus other $C Y P 2 D 6^{*} 2$ alleles. There is evidence that the $C Y P 2 D 6^{*} 2$ alleles (except $C Y P 2 D 6^{*} 2 A$ ) have reduced function, although this is somewhat substrate-dependent. ${ }^{41-44}$ However, the c. $1584 \mathrm{C}>\mathrm{G}$ polymorphism found in $C Y P 2 D 6 * 2 A$ increases protein production, possibly through increased induction, which compensates for the reduced function caused by the other polymorphisms found in the $C Y P 2 D 6^{*} 2$ alleles, resulting in a function similar to and possibly greater than that of CYP2D $6 * 1 .^{45,46}$

The classification used in our labs for CYP2D6 was as follows: ultrarapid, extensive to ultrarapid, extensive, intermediate to extensive, intermediate, poor to intermediate, and poor. For analysis, these categories were further grouped into three phenotype categories: poor (poor and poor to intermediate), intermediate to extensive (intermediate, intermediate to extensive, and extensive), or ultrarapid (extensive to ultrarapid and ultrarapid) $C Y P 2 D 6$ metabolizers.

\section{Outcomes}

All electronic health records (EHRs) were reviewed by a single author (JLS) for indications of poor pain control (e.g., note indicating need to change dose or type of drug due to pain) or adverse symptoms related to medication use (e.g., vomiting, nausea, rash, itching, throat swelling, or other indication of adverse reaction attributed to the prescription). All records were reviewed without knowledge of the patient phenotypes.

\section{CYP2D6 inhibitors}

Concurrent use of CYP2D6 inhibitor medications may significantly alter the results of studies that examine outcomes related to the use of opioid medications, because these inhibitors may decrease or prevent the conversion of opioid medications to morphine. Therefore, we identified all patients who were prescribed a medication that was a strong or moderately strong inhibitor of CYP2D6. A list of inhibitors that was considered for this study is shown in Table S3. Because most of these prescriptions are intended to be taken long term, we considered persons exposed to a CYP2D6 inhibitor if the prescription occurred within 1 year prior to the opioid prescription.

\section{Analyses}

The frequencies of demographic and medication characteristics were summarized using counts and percentages. Associations between the three assigned phenotype groups (poor, intermediate to extensive, and ultrarapid) and outcomes (poor pain control, adverse reaction or either) were assessed using Chi-square tests for categorical variables. Logistic regression was used to model associations between phenotype group and each outcome and is reported as odds ratios (ORs) and 95\% CIs. Multivariable models were used to adjust for age and sex.

Limited sample sizes precluded adjustment for additional variables. However, in separate models, we also adjusted for the type of prescription (codeine or tramadol vs oxycodone or hydrocodone) and the total number of prescriptions in the time frame. Results were similar to models adjusted for age and sex; therefore, only age- and sex-adjusted results are reported.

Finally, we conducted two additional sensitivity analyses. First, to account for potential interactions with medications that are known to inhibit CYP2D6, we excluded all persons who were prescribed a CYP2D6 inhibitor. Second, we repeated our analyses excluding all persons with a $* 2 \mathrm{~A} / * 2 \mathrm{~A}$ genotype, since, as described above, there is some controversy regarding whether the $* 2 \mathrm{~A}$ allele truly has increased activity.

\section{Results}

Participant characteristics, including indications and types of prescriptions, are shown in Table 1. A total of 257 (25\%) 
Table I Characteristics of study participants in the RIGHT protocol that received at least one opioid prescription between July 01,2013 and June 30, 2015

\begin{tabular}{|c|c|c|}
\hline Characteristic & $\mathbf{N}$ & $\%$ \\
\hline \multicolumn{3}{|l|}{ Sex } \\
\hline Male & 113 & 44 \\
\hline Female & 144 & 56 \\
\hline \multicolumn{3}{|l|}{ Age } \\
\hline $40-64$ & 215 & 84 \\
\hline $65+$ & 42 & 16 \\
\hline \multicolumn{3}{|l|}{ Race } \\
\hline White & 247 & 96 \\
\hline Black & I & 0.4 \\
\hline Asian/Pacific Islander & 3 & I \\
\hline Other & 6 & 2 \\
\hline \multicolumn{3}{|l|}{ Ethnicity } \\
\hline Hispanic & 2 & 1 \\
\hline Non-Hispanic & 255 & 99 \\
\hline \multicolumn{3}{|l|}{ CYP2D6 phenotype } \\
\hline Poor metabolizer & 40 & 15 \\
\hline Intermediate to extensive metabolizer & 146 & 57 \\
\hline Ultrarapid metabolizer & 7I & 28 \\
\hline \multicolumn{3}{|l|}{ Number of prescriptions ${ }^{a}$} \\
\hline Codeine & 18 & 7 \\
\hline Oxycodone & 167 & 65 \\
\hline Hydrocodone & 63 & 25 \\
\hline Tramadol & 109 & 42 \\
\hline \multicolumn{3}{|c|}{ Total number of prescriptions during the 2-year period } \\
\hline $\mathrm{I}$ & 122 & 48 \\
\hline 2 & 60 & 23 \\
\hline 3 & 20 & 8 \\
\hline $4+$ & 55 & 21 \\
\hline \multicolumn{3}{|l|}{ Reason for prescription ${ }^{b}$} \\
\hline Surgery/procedure & $|3|$ & 51 \\
\hline Trauma & 21 & 8 \\
\hline Chronic joint/back pain & 75 & 29 \\
\hline Other & 52 & 20 \\
\hline Prescribed a CYP2D6 inhibitor & 38 & 15 \\
\hline
\end{tabular}

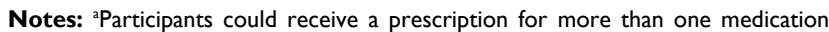
during the time frame. 'Participants could have multiple reasons for a single prescription.

patients received at least one opioid prescription in the 2 year-time period. More women than men received a prescription, and most of those who received a prescription were between 40 and 64 years of age. Among those with an opioid prescription, $57 \%$ had an intermediate to extensive metabolizer CYP2D6 phenotype, whereas $43 \%$ had either a poor or ultrarapid phenotype (Table 1). In addition, over half $(51 \%)$ received their opioid prescription to control pain following surgery or a medical procedure, and $65 \%$ of the prescriptions were for oxycodone. Finally, 38 persons were also prescribed a medication that inhibits $C Y P 2 D 6$ within 1 year of the opioid prescription. Of these persons, 5 were poor metabolizers, 22 were intermediate to extensive metabolizers, and 11 were ultrarapid metabolizers (Table 1). Among the patients with poor pain control, health care providers increased the dose of the medication or changed the medication type in 19 patients (63\%), and recommended further rest or patience for 3 additional patients (10\%). In the remainder of the cases, a note indicating that the patient was experiencing poor pain control was present in the record, but the health care provider response was not recorded. Among patients with adverse reactions, most were related to gastrointestinal upset $(21 ; 55 \%)$. Itching, rash, or throat swelling were present in $9(24 \%)$ patients, while the remaining patients had a mix of nonspecific reactions (e.g., "extreme withdrawal", dizziness).

Initial analyses indicated that $35 \%$ of poor metabolizers and $34 \%$ of ultrarapid metabolizers experienced either poor pain control or an adverse symptom following an opioid prescription compared to $18 \%$ of intermediate to extensive metabolizers (Table 2). Overall, 19 (17\%) participants with a poor or ultrarapid phenotype experienced poor pain control, compared to $11(8 \%)$ intermediate to extensive metabolizers $(p=0.05)$. Similarly, $21(19 \%)$ patients with a poor or ultrarapid phenotype experienced an adverse symptom related to the prescribed medication compared to $17(12 \%)$ intermediate to extensive metabolizers $(p=0.25$; Table 2). These results were similar in sensitivity analyses that accounted for persons who were prescribed a CYP2D6 inhibitor (Table 2). However, associations with poor pain control were attenuated, while associations with adverse reactions were strengthened. Because poor and ultrarapid metabolizers experienced similar rates of both poor pain control and adverse reactions in all analyses, these patients were grouped together into an "extreme phenotype" category for additional analyses.

After adjusting for age and sex, patients with an extreme phenotype were 2.6 times more likely to experience poor pain control related to the prescription and 1.8 times more likely to experience an adverse symptom compared to intermediate to extensive metabolizers (Table 3). However, the association between extreme phenotype and adverse reaction was not statistically significant (OR: 1.77; 95\% CI: 0.87, 3.57). Overall, patients with an extreme phenotype were 2.4 times more likely to experience any problem following an opioid prescription than patients with an intermediate to extensive phenotype (OR: 2.40 ; 95\% CI: 1.35, 4.28).

After accounting for persons that were also prescribed a CYP2D6 inhibitor, the association between extreme phenotype and poor pain control was attenuated and no longer significant. However, the association between extreme phenotype and adverse reaction strengthened and attained significance (Table 3). The associations between an extreme 
Table 2 Associations between CYP2D6 metabolizer status and poor pain control or other adverse reactions following prescription of an opioid medication

\begin{tabular}{|c|c|c|c|c|c|c|}
\hline \multirow[t]{2}{*}{ CYP2D6 metabolizer status } & \multicolumn{2}{|c|}{ Poor pain control } & \multicolumn{2}{|c|}{ Adverse reaction } & \multicolumn{2}{|c|}{ Any problem ${ }^{a}$} \\
\hline & $\mathbf{n}$ & $\%$ & $\mathbf{n}$ & $\%$ & $\mathbf{n}$ & $\%$ \\
\hline \multicolumn{7}{|l|}{ All persons } \\
\hline Poor $(\mathrm{N}=40)$ & 8 & 20 & 7 & 18 & 14 & 35 \\
\hline Intermediate to extensive $(\mathrm{N}=\mid 46)$ & 11 & 8 & 17 & 12 & 26 & 18 \\
\hline Ultrarapid (N=7I) & 11 & 16 & 14 & 20 & 24 & 34 \\
\hline Chi-square $p$-value & 0.05 & & 0.25 & & 0.01 & \\
\hline \multicolumn{7}{|c|}{ Excluding those prescribed a CYP2D6 inhibitor } \\
\hline Poor $(\mathrm{N}=35)$ & 5 & 14 & 6 & 17 & 11 & 31 \\
\hline Intermediate to extensive $(\mathrm{N}=124)$ & 9 & 7 & 11 & 9 & 19 & 15 \\
\hline Ultrarapid $(\mathrm{N}=60)$ & 8 & 13 & 13 & 22 & 20 & 33 \\
\hline Chi-square $p$-value & 0.29 & & 0.05 & & 0.01 & \\
\hline
\end{tabular}

Notes: aPatients could experience both poor pain control and an adverse reaction. If so, they were only counted once as having "any problem".

Table 3 Adjusted odds of poor pain control or other adverse reaction following the prescription of an opioid medication among poor/ ultrarapid CYP2D6 metabolizers compared to intermediate to extensive metabolizers

\begin{tabular}{|c|c|c|c|c|c|c|c|c|c|}
\hline \multirow[t]{2}{*}{ CYP2D6 metabolizer status } & \multicolumn{3}{|c|}{ Poor pain control ${ }^{\mathbf{a}}$} & \multicolumn{3}{|c|}{ Adverse reaction $^{\mathrm{a}}$} & \multicolumn{3}{|c|}{ Any problem ${ }^{a}$} \\
\hline & OR & $95 \% \mathrm{Cl}$ & $p$-value & OR & $95 \% \mathrm{Cl}$ & p-value & OR & $95 \% \mathrm{Cl}$ & $p$-value \\
\hline All patients & 2.63 & $1.19,5.83$ & 0.02 & 1.77 & $0.87,3.57$ & 0.11 & 2.40 & $1.35,4.28$ & 0.003 \\
\hline Excluded patients prescribed a CYP2D6 inhibitor & 2.15 & $0.87,5.32$ & 0.10 & 2.53 & $1.12,5.70$ & 0.03 & 2.68 & $1.39,5.17$ & 0.003 \\
\hline $\begin{array}{l}\text { Excluded patients prescribed a CYP2D6 inhibitor } \\
\text { and a } * 2 \mathrm{~A} / * 2 \mathrm{~A} \text { genotype }\end{array}$ & 1.74 & $0.65,4.66$ & 0.27 & 2.51 & $1.07,5.90$ & 0.03 & 2.49 & $1.25,4.95$ & 0.01 \\
\hline $\begin{array}{l}\text { Patients prescribed only oxycodone or } \\
\text { hydrocodone, excluding patients prescribed a } \\
\text { CYP2D6 inhibitor and a } * 2 A / 2 A \text { genotype }\end{array}$ & 1.71 & $0.55,5.30$ & 0.35 & 2.08 & $0.67,6.49$ & 0.21 & 2.23 & $0.94,5.25$ & 0.07 \\
\hline
\end{tabular}

Notes: aResults are compared to intermediate to extensive CYP2D6 metabolizers and are adjusted for age and sex.

phenotype and any problem were unchanged. Additional exclusion of persons with a $* 2 \mathrm{~A} / * 2 \mathrm{~A}$ genotype did not significantly alter the results (Table 3 ). Finally, limiting the analysis to only persons prescribed oxycodone and hydrocodone further attenuated the associations; however, results were in the same direction, and were consistent with overall results (Table 3 ).

\section{Discussion}

We found that patients with either a poor or an ultrarapid CYP2D6 phenotype were more likely to have an EHR note indicating poor pain relief and to experience more adverse medication effects compared to intermediate to extensive metabolizers. Overall, approximately one-third of patients with an extreme CYP2D6 phenotype experienced problems related to opioid use compared to $\sim 20 \%$ of patients with an intermediate to extensive phenotype.

Previous studies have shown that persons with a poor metabolizer CYP2D6 phenotype are less likely to experience pain relief compared to persons with normal CYP2D6 phenotypes, particularly following a codeine or tramadol prescription. ${ }^{19-25}$ However, data showing a poor analgesic effect after use of hydrocodone or oxycodone among poor CYP2D6 metabolizers have been less consistent. ${ }^{26-30}$ Our data indicate that persons with a poor metabolizer status were also more likely to have a report of poor pain control in their EHR, and these results are consistent with previous studies.

Research on clinical outcomes in ultrarapid metabolizers is lacking. Expanding the state of the literature, our results indicate that ultrarapid metabolizers also had reports of poor pain control in their records at approximately the same rate as the poor metabolizers. These patients are expected to be at higher risk of drug intoxication or other adverse events, but the lack of pain control has not been emphasized previously. It is not clear why these patients might report lower levels of pain control. However, it is possible that these patients may not tolerate these prescriptions due to a higher rate of adverse symptoms, and are more likely to stop taking these medications. We note, however, that associations between either poor or ultrarapid status and poor pain control were not statistically significant after accounting for the prescription of CYP2D6 inhibitor medications, but poor pain control was still present more often in those with poor or ultrarapid metabolizer status compared to those with an intermediate to extensive 
metabolizer status. Our small number of outcomes makes these estimates unstable, and results must be confirmed in a larger population.

We also found that patients with either a poor or ultrarapid phenotype were more likely to experience an adverse drug effect such as nausea or vomiting compared to those with an intermediate to extensive phenotype. These associations strengthened after accounting for the prescription of CYP2D6 inhibitor medications. Ultrarapid metabolizers have previously been shown to be at higher risk of life-threatening reactions compared to normal metabolizers when given standard doses of these medications. ${ }^{47}$ However, data showing associations between ultrarapid metabolizer status and less severe reactions are limited. Data showing associations between poor metabolizer status and adverse drug reactions are also limited, but one small study with 18 participants suggested that metabolizer status was not associated with adverse reactions to hydrocodone. ${ }^{19}$ Our results suggest that persons with an extreme phenotype are more likely to experience adverse drug effects compared to intermediate to extensive metabolizers, and this effect was statistically significant after accounting for the use of CYP2D6 inhibitor medications.

\section{Strengths and limitations}

Strengths of our study included our relatively large sample size for studies that have examined associations between CYP2D6 phenotypes and response to opioid analgesics. In addition, we had access to patient EHR data and were able to examine these associations in the context of real-life medical care, and were able to examine multiple drugs simultaneously.

Significant limitations of our study include a high potential for misclassification. Specifically, we relied on complete reporting of pain response and adverse drug events through the EHR. Such data may be incompletely reported by patients and recorded by the health care practitioners, and we may have missed adverse events that actually occurred. If there is differential reporting or recording of adverse outcomes by $C Y P 2 D 6$ phenotype, our results could be biased. First, if persons with an extreme phenotype were less likely to have an adverse outcome reported in their record than those with a normal phenotype, our results will underestimate the true association between extreme phenotype and adverse outcomes. If persons with an extreme phenotype were more likely to have an adverse outcome reported compared to those with a normal phenotype, our results will overestimate the true association. However, both patients and health care providers were unaware of the patient's phenotype at the time of the prescriptions. Therefore, we expect that the most likely scenario is that adverse events are consistently underreported in the medical records regardless of patient phenotype. If such data are incompletely recorded regardless of phenotype, the result will be an overall reduction in the number of possible outcomes, and our results would be less likely to reach statistical significance, but would not be biased. In fact, it is somewhat surprising that we observed statistically significant associations.

We also lack data regarding medication compliance. If compliance and adverse drug events were reported differentially by $C Y P 2 D 6$ phenotype, our results may be biased. We expect that persons with a past history of opioid use that they attributed to an adverse event are less likely to actually take their prescription compared to persons who had no problems with the prescription. We also expect that persons with an extreme CYP2D6 phenotype are more likely in general to have had a poor experience with opioid medications, and these persons would be less likely to take their prescribed medications. Failing to take an opioid may result in poor pain control, and this could account for the unexpected finding that persons with an ultrarapid phenotype seemed to experience poor pain control at about the same rate as poor metabolizers.

Finally, the Luminex kit used for genotyping in this study did not include some alleles that could change the phenotype for some of our participants. Chiefly, the *2A allele (an increased activity allele) harbors some $* 35$ alleles (a normal activity allele). Therefore, some of the $* 2 \mathrm{~A}$ alleles may have actually been $* 35$ alleles, and these persons were incorrectly classified as ultrarapid metabolizers rather than as intermediate to extensive metabolizers. Therefore, we excluded all persons with $* 2 \mathrm{~A} / * 2 \mathrm{~A}$ genotypes in a sensitivity analysis. Results were very similar to including these persons, suggesting that misclassification was not extensive. Future studies, however, will include typing that allows for the detection of $* 35$ alleles. Similarly, intermediate to extensive CYP2D6 metabolizers may have highly variable phenotypes due to nongenetic factors. Therefore, some of these persons are likely incorrectly classified as not having an extreme phenotype. If these persons experience adverse outcomes at a lower rate than persons in the "extreme" phenotype groups, then our results are an overestimate of the true association. However, if these persons experience adverse outcomes at the same or higher rate as our "extreme" phenotype groups, our results are an underestimate of the true association.

Many of the pain medications identified in this study were prescribed in conjunction with other medications, making it difficult to definitively attribute some events to a single prescription. We also relied on prescription information and do 
not know how much or how often the medication was taken. We did account for the prescription of strong or moderately strong CYP2D6 inhibitor medications, and our results were consistent regardless of whether persons taking these medications were excluded or reclassified. Accounting for these medications substantially strengthened associations between extreme metabolizer status and adverse reactions, indicating that it is important to account for use of these medications in future analyses.

Oxycodone and hydrocodone are predominantly metabolized by $C Y P 3 A 4$, and variation in $C Y P 3 A 4$ function may significantly impact an individual's response to these medications (Yiannakopoulou, 2015 \#104). We conducted a subanalysis of persons that were only prescribed oxycodone or hydrocodone, and results were attenuated compared to the overall results (as expected, due to smaller sample sizes). However, all results were still in the same direction, and point estimates were of a similar magnitude, suggesting that variation in CYP2D6 is still an important factor to consider when considering response to oxycodone and hydrocodone. Finally, while the study sample size was relatively large, the number of outcomes was small, and estimates may not be stable.

\section{Conclusion}

In summary, $34 \%$ of patients with an extreme CYP2D6 phenotype experienced an adverse outcome when prescribed codeine, tramadol, oxycodone, or hydrocodone. Opioid medications are frequently prescribed for pain relief, and $\sim 39 \%$ of the US population is expected to carry an extreme phenotype (as defined by our study), suggesting that the population-level impact of these gene-drug interactions could be substantial.

\section{Acknowledgments}

The authors thank Ms. Robin Adams for her assistance in typing and formatting the manuscript, and Ms. Ruoxiang Jiang for analytic support and review of this manuscript. The authors also thank Dr. Muhamad Elrashidi and Ms. Carolyn Roer Vitek for expertise regarding the likely impact of pharmacogenomics-guided opioid prescribing on primary care patients and physicians, and for their review and revision of this manuscript.

This work was supported in part by Mayo Clinic Center for Individualized Medicine, the Robert D. and Patricia E. Kern Center for the Science of Health Care Delivery, National Institutes of Health grants U19 GM61388 (The Pharmacogenomics Research Network), R01 GM28157, U01 HG005137, R01 CA138461, R01 AG034676 (The
Rochester Epidemiology Project), and U01 HG06379 and U01 HG06379 Supplement (The Electronic Medical Record and Genomics [eMERGE] Network).

\section{Disclosure}

John L Black III has licensed intellectual property to the companies AssureX Health and OneOme. In addition, he has stock ownership in OneOme. The other authors report no conflicts of interest in this work.

\section{References}

1. Dowell D, Haegerich TM, Chou R. CDC guideline for prescribing opioids for chronic pain - United States, 2016. JAMA. 2016;315(15): 1624-1645.

2. Thorson D, Biewen P, Bonte B, et al. Acute Pain Assessment and Opioid Prescribing Protocol. Bloomington, MN: the Institute for Clinical Systems Improvement (ICSI); 2014

3. Paulozzi LJ, Mack KA, Hockenberry JM. Vital signs: variation among States in prescribing of opioid pain relievers and benzodiazepines United States, 2012. MMWR Morb Mortal Wkly Rep. 2014;63(26): 563-568.

4. Frenk SM, Porter KS, Paulozzi LJ. Prescription opioid analgesic use among adults: United States, 1999-2012. NCHS Data Brief. 2015(189):1-8.

5. Steinman MA, Komaiko KD, Fung KZ, Ritchie CS. Use of opioids and other analgesics by older adults in the United States, 1999-2010. Pain Med. 2015;16(2):319-327.

6. Abdel Shaheed C, Maher CG, Williams KA, Day R, McLachlan AJ. Efficacy, tolerability, and dose-dependent effects of opioid analgesics for low back pain: a systematic review and meta-analysis. JAMA Intern Med. 2016;176(7):958-968.

7. Riley JL 3rd, Hastie BA. Individual differences in opioid efficacy for chronic noncancer pain. Clin J Pain. 2008;24(6):509-520.

8. Kalso E, Edwards JE, Moore RA, McQuay HJ. Opioids in chronic noncancer pain: systematic review of efficacy and safety. Pain. 2004;112(3): 372-380.

9. Breivik H, Collett B, Ventafridda V, Cohen R, Gallacher D. Survey of chronic pain in Europe: prevalence, impact on daily life, and treatment. Eur J Pain. 2006;10(4):287-333.

10. Chou R, Clark E, Helfand M. Comparative efficacy and safety of longacting oral opioids for chronic non-cancer pain: a systematic review. J Pain Symptom Manage. 2003;26(5):1026-1048.

11. XuY, Johnson A. Opioid therapy pharmacogenomics for noncancer pain: efficacy, adverse events, and costs. Pain Res Treat. 2013;2013:943014.

12. Ting S, Schug S. The pharmacogenomics of pain management: prospects for personalized medicine. J Pain Res. 2016;9:49-56.

13. Crews KR, Gaedigk A, Dunnenberger HM, et al. Clinical pharmacogenetics implementation consortium guidelines for cytochrome P450 2D6 genotype and codeine therapy: 2014 update. Clin Pharmacol Ther. 2014;95(4):376-382.

14. Kadiev E, Patel V, Rad P, et al. Role of pharmacogenetics in variable response to drugs: focus on opioids. Expert Opin Drug Metab Toxicol. 2008;4(1):77-91.

15. Lotsch J, Skarke C, Liefhold J, Geisslinger G. Genetic predictors of the clinical response to opioid analgesics: clinical utility and future perspectives. Clin Pharmacokinet. 2004;43(14):983-1013.

16. Zahari Z, Ismail R. Influence of Cytochrome P450, Family 2, Subfamily D, Polypeptide 6 (CYP2D6) polymorphisms on pain sensitivity and clinical response to weak opioid analgesics. Drug Metab Pharmacokinet. 2014;29(1):29-43.

17. Candiotti KA, Yang Z, Rodriguez Y, et al. The impact of CYP2D6 genetic polymorphisms on postoperative morphine consumption. Pain Med. 2009;10(5):799-805. 
18. Yiannakopoulou E. Pharmacogenomics and opioid analgesics: clinical implications. Int J Genomics. 2015;2015:368979.

19. Eckhardt K, Li S, Ammon S, Schanzle G, Mikus G, Eichelbaum M. Same incidence of adverse drug events after codeine administration irrespective of the genetically determined differences in morphine formation. Pain. 1998;76(1-2):27-33.

20. Lotsch J, Rohrbacher M, Schmidt H, Doehring A, Brockmoller J, Geisslinger G. Can extremely low or high morphine formation from codeine be predicted prior to therapy initiation? Pain. 2009;144(1-2): $119-124$.

21. Slanar O, Dupal P, Matouskova O, Vondrackova H, Pafko P, Perlik F. Tramadol efficacy in patients with postoperative pain in relation to CYP2D6 and MDR1 polymorphisms. Bratisl Lek Listy. 2012;113(3):152-155.

22. Stamer UM, Musshoff F, Kobilay M, Madea B, Hoeft A, Stuber F. Concentrations of tramadol and O-desmethyltramadol enantiomers in different CYP2D6 genotypes. Clin Pharmacol Ther. 2007;82(1):41-47.

23. Poulsen L, Arendt-Nielsen L, Brosen K, Sindrup SH. The hypoalgesic effect of tramadol in relation to CYP2D6. Clin Pharmacol Ther. 1996;60(6):636-644.

24. Stamer UM, Lehnen K, Hothker F, et al. Impact of CYP2D6 genotype on postoperative tramadol analgesia. Pain. 2003;105(1-2):231-238.

25. Wang G, Zhang H, He F, Fang X. Effect of the CYP2D6*10 C188T polymorphism on postoperative tramadol analgesia in a Chinese population. Eur J Clin Pharmacol. 2006;62(11):927-931.

26. Otton SV, Schadel M, Cheung SW, Kaplan HL, Busto UE, Sellers EM. CYP2D6 phenotype determines the metabolic conversion of hydrocodone to hydromorphone. Clin Pharmacol Ther. 1993;54(5):463-472.

27. Samer CF, Daali Y, Wagner M, et al. Genetic polymorphisms and drug interactions modulating CYP2D6 and CYP3A activities have a major effect on oxycodone analgesic efficacy and safety. Br J Pharmacol. 2010;160(4):919-930.

28. Zwisler ST, Enggaard TP, Mikkelsen S, Brosen K, Sindrup SH. Impact of the CYP2D6 genotype on post-operative intravenous oxycodone analgesia. Acta Anaesthesiol Scand. 2010;54(2):232-240.

29. Zwisler ST, Enggaard TP, Noehr-Jensen L, et al. The hypoalgesic effect of oxycodone in human experimental pain models in relation to the CYP2D6 oxidation polymorphism. Basic Clin Pharmacol Toxicol. 2009;104(4):335-344.

30. Andreassen TN, Eftedal I, Klepstad P, et al. Do CYP2D6 genotypes reflect oxycodone requirements for cancer patients treated for cancer pain? A cross-sectional multicentre study. Eur J Clin Pharmacol. 2012;68(1):55-64.

31. Bielinski SJ, Olson JE, Pathak J, et al. Preemptive genotyping for personalized medicine: design of the right drug, right dose, right timeusing genomic data to individualize treatment protocol. Mayo Clin Proc. 2014;89(1):25-33.

32. Olson JE, Ryu E, Johnson KJ, et al. The Mayo Clinic Biobank: a building block for individualized medicine. Mayo Clin Proc. 2013;88(9): 952-962.

33. St Sauver JL, Grossardt BR, Yawn BP, et al. Data resource profile: the Rochester Epidemiology Project (REP) medical records-linkage system. Int J Epidemiol. 2012;41(6):1614-1624.
34. Zhong W, Maradit-Kremers H, St Sauver JL, et al. Age and sex patterns of drug prescribing in a defined American population. Mayo Clin Proc. 2013;88(7):697-707.

35. Black JL, 3rd, Walker DL, O'Kane DJ, Harmandayan M. Frequency of undetected CYP2D6 hybrid genes in clinical samples: impact on phenotype prediction. Drug Metab Dispos. 2012;40(1):111-119.

36. JiY, Skierka JM, Blommel JH, et al. Preemptive pharmacogenomic testing for precision medicine: a comprehensive analysis of five actionable pharmacogenomic genes using next-generation DNA sequencing and a customized CYP2D6 genotyping cascade. J Mol Diagn. 2016;18(3):438-445.

37. Ingelman-Sundberg M. Genetic polymorphisms of cytochrome P450 2D6 (CYP2D6): clinical consequences, evolutionary aspects and functional diversity. Pharmacogenomics J. 2005;5(1):6-13.

38. Kirchheiner J, Nickchen K, Bauer M, et al. Pharmacogenetics of antidepressants and antipsychotics: the contribution of allelic variations to the phenotype of drug response. Mol Psychiatry. 2004;9(5):442-473.

39. Steimer W, Zopf K, von Amelunxen S, et al. Allele-specific change of concentration and functional gene dose for the prediction of steady-state serum concentrations of amitriptyline and nortriptyline in CYP2C19 and CYP2D6 extensive and intermediate metabolizers. Clin Chem. 2004;50(9):1623-1633.

40. Gaedigk A, Simon SD, Pearce RE, Bradford LD, Kennedy MJ, Leeder JS. The CYP2D6 activity score: translating genotype information into a qualitative measure of phenotype. Clin Pharmacol Ther. 2008;83(2):234-242.

41. Raimundo S, Toscano C, Klein K, et al. A novel intronic mutation, 2988G $>$ A, with high predictivity for impaired function of cytochrome P450 2D6 in white subjects. Clin Pharmacol Ther. 2004;76(2):128-138.

42. Bapiro TE, Hasler JA, Ridderstrom M, Masimirembwa CM. The molecular and enzyme kinetic basis for the diminished activity of the cytochrome P450 2D6.17 (CYP2D6.17) variant. Potential implications for CYP2D6 phenotyping studies and the clinical use of CYP2D6 substrate drugs in some African populations. Biochem Pharmacol. 2002;64(9):1387-1398.

43. Yu A, Kneller BM, Rettie AE, Haining RL. Expression, purification, biochemical characterization, and comparative function of human cytochrome P450 2D6.1, 2D6.2, 2D6.10, and 2D6.17 allelic isoforms. J Pharmacol Exp Ther. 2002;303(3):1291-1300.

44. Abduljalil K, Frank D, Gaedigk A, et al. Assessment of activity levels for CYP2D6*1, CYP2D6*2, and CYP2D6*41 genes by population pharmacokinetics of dextromethorphan. Clin Pharmacol Ther. 2010;88(5):643-651.

45. Lovlie R, Daly AK, Matre GE, Molven A, Steen VM. Polymorphisms in CYP2D6 duplication-negative individuals with the ultrarapid metabolizer phenotype: a role for the CYP2D6*35 allele in ultrarapid metabolism? Pharmacogenetics. 2001;11(1):45-55.

46. Zanger UM, Fischer J, Raimundo S, et al. Comprehensive analysis of the genetic factors determining expression and function of hepatic CYP2D6. Pharmacogenetics. 2001;11(7):573-585.

47. Haufroid V, Hantson P. CYP2D6 genetic polymorphisms and their relevance for poisoning due to amfetamines, opioid analgesics and antidepressants. Clin Toxicol (Phila). 2015;53(6):501-510. 


\section{Supplementary materials}

Table SI Ingredient names and RxNorm codes of opioid prescription medications included in the study

\begin{tabular}{|c|c|}
\hline $\begin{array}{l}\text { RxNorm } \\
\text { code }\end{array}$ & Ingredient name \\
\hline 821601 & Acetaminophen/Aspirin/Caffeine/Codeine \\
\hline 689552 & Acetaminophen/Aspirin/Caffeine/Codeine/salicylamide \\
\hline 689555 & Acetaminophen/Aspirin/Codeine \\
\hline 689561 & Acetaminophen/butalbital/Caffeine/Codeine \\
\hline 689563 & Acetaminophen/butalbital/Codeine \\
\hline 814657 & Acetaminophen/Caffeine/Codeine \\
\hline 817430 & Acetaminophen/Caffeine/Codeine/Meprobamate \\
\hline 689568 & Acetaminophen/Caffeine/Codeine/salicylamide \\
\hline 817579 & Acetaminophen/Codeine \\
\hline 817356 & Acetaminophen/Codeine/lbuprofen \\
\hline 1007238 & Aconite/Codeine/Erysimum preparation \\
\hline 690996 & Aluminum Hydroxide/Aspirin/Codeine/Magnesium Hydroxide \\
\hline 214237 & anhydrous calcium iodide/Codeine \\
\hline 214160 & Aspirin/butalbital/Caffeine/Codeine \\
\hline 689511 & Aspirin/Caffeine/Codeine \\
\hline 689522 & Aspirin/Carisoprodol/Codeine \\
\hline 135095 & Aspirin/Codeine \\
\hline 1008493 & Benzoate/Codeine \\
\hline 1008060 & Butalbital/Caffeine/Codeine \\
\hline 691032 & Calcium iodide/Codeine \\
\hline 2670 & Codeine \\
\hline 1008110 & Codeine/Diclofenac \\
\hline 1008954 & Codeine/Erysimum preparation \\
\hline 1007477 & Codeine/Ethylmorphine \\
\hline 710303 & Codeine/lbuprofen \\
\hline 214443 & Codeine/iodinated glycerol \\
\hline 729517 & Codeine/Kaolin \\
\hline 690089 & Codeine/Papaverine \\
\hline 1007293 & Codeine/Potassium \\
\hline 690096 & Codeine/potassium citrate \\
\hline 1007204 & Codeine/propyphenazone \\
\hline 690101 & Codeine/Pyrilamine \\
\hline 689569 & Acetaminophen/Caffeine/dihydrocodeine \\
\hline 151196 & Acetaminophen/dihydrocodeine \\
\hline 689783 & Acetaminophen/dihydrocodeine/salicylamide \\
\hline 689512 & Aspirin/Caffeine/dihydrocodeine \\
\hline 23088 & Dihydrocodeine \\
\hline 214183 & Acetaminophen/Oxycodone \\
\hline 214256 & Aspirin/Oxycodone \\
\hline 484259 & Ibuprofen/Oxycodone \\
\hline 1545902 & Naloxone/Oxycodone \\
\hline 7804 & Oxycodone \\
\hline 689553 & Acetaminophen/Aspirin/Caffeine/Hydrocodone \\
\hline 689562 & Acetaminophen/butalbital/Caffeine/Hydrocodone \\
\hline 214182 & Acetaminophen/Hydrocodone \\
\hline 689515 & Aspirin/Caffeine/Hydrocodone \\
\hline 214253 & Aspirin/Hydrocodone \\
\hline 5489 & Hydrocodone \\
\hline 214627 & Hydrocodone/lbuprofen \\
\hline 352362 & Acetaminophen/Tramadol \\
\hline 10689 & Tramadol \\
\hline
\end{tabular}


Table S2 Ingredient name and count of additional prescriptions received on the same day as the opioid prescription

\begin{tabular}{|c|c|}
\hline Ingredient RxNorm description & $\mathbf{N}$ \\
\hline Acetominophen & 8 \\
\hline Albuterol & 2 \\
\hline Amoxicillin or Amoxicillin/Clavulanate & 7 \\
\hline Aspirin & 3 \\
\hline Azithromycin & 2 \\
\hline Bacitracin & 1 \\
\hline Benzonatate & 1 \\
\hline Cefadroxil & $\mathrm{I}$ \\
\hline Cefdinir & 1 \\
\hline Celecoxib & 2 \\
\hline Cephalexin & 9 \\
\hline Ciprofloxacin/Dexamethasone & 1 \\
\hline Ciprofloxacin opthalmic preparation & 10 \\
\hline Clindamycin & 1 \\
\hline Colchicine & 1 \\
\hline Cyclobenzaprine & 8 \\
\hline Daptomycin & 1 \\
\hline Dexamethasone & 3 \\
\hline Diazepam & 5 \\
\hline Diclofenac & 2 \\
\hline Dicloxacillin & 1 \\
\hline Diflunisal & 1 \\
\hline Docosanol & $\mathrm{I}$ \\
\hline Docusate & 17 \\
\hline Docusate/Sennosides & 16 \\
\hline Enoxaparin & 4 \\
\hline Erythromycin Base & 3 \\
\hline Estrogens, conjugated & 3 \\
\hline Fenofibrate & I \\
\hline Fentanyl & 1 \\
\hline Fexofenadine & 1 \\
\hline Fluoxetine & 1 \\
\hline Furosemide & 2 \\
\hline Gabapentin & $\mathrm{I}$ \\
\hline Haloperidol & 1 \\
\hline Hydromorphone & 1 \\
\hline Indomethacin & 2 \\
\hline Insulin Aspart & 1 \\
\hline Insulin glargine, hum rec analog & 1 \\
\hline Ketoconazole & 1 \\
\hline$L-$ Thyroxine & 2 \\
\hline Levofloxacin & $\mathrm{I}$ \\
\hline Lidocaine & 2 \\
\hline Lisinopril & 1 \\
\hline Loratadine/Pseudoephedrine & I \\
\hline Lorazepam & 2 \\
\hline Methylphenidate & 1 \\
\hline Metoprolol & $\mathrm{I}$ \\
\hline Metronidazole & 2 \\
\hline Miconazole & 1 \\
\hline Minocycline & 1 \\
\hline Montelukast & 2 \\
\hline Morphine & 2 \\
\hline Moxifloxacin & 1 \\
\hline Naproxen & 3 \\
\hline Nortriptyline & 1 \\
\hline Omeprazole & 3 \\
\hline
\end{tabular}


Table S2 (Continued)

\begin{tabular}{ll}
\hline Ingredient RxNorm description & $\mathbf{N}$ \\
\hline Ondansetron & $\mathrm{II}$ \\
Oxybutynin & 5 \\
Pantoprazole & $\mathrm{I}$ \\
Phenazopyridine & $\mathrm{I}$ \\
Potassium chloride & $\mathrm{I}$ \\
Prednisolone & $\mathrm{I}$ \\
Promethazine & 2 \\
Psyllium & $\mathrm{I}$ \\
Rifampin & $\mathrm{I}$ \\
Rivaroxaban & $\mathrm{I}$ \\
Sennoside A \& B & 2 \\
Sertraline & 2 \\
Sildenafil & $\mathrm{I}$ \\
Sulfamethoxazole/Trimethoprim & $\mathrm{I}$ \\
Tadalafil & $\mathrm{I}$ \\
Tamsulosin & 8 \\
Trazodone & $\mathrm{I}$ \\
Triamcinolone & 2 \\
Vancomycin & $\mathrm{I}$ \\
Warfarin & 3 \\
Zolpidem & $\mathrm{I}$ \\
\hline
\end{tabular}

Table S3 Ingredient names and RxNorm codes of strong or moderately strong CYP2D6 inhibitors included in the study

\begin{tabular}{ll}
\hline RxNorm code & Ingredient name \\
\hline 42347 & Bupropion \\
1551467 & Bupropion/Naltrexone \\
407990 & Cinacalcet \\
4493 & Fluoxetine \\
611247 & Fluoxetine/olanzapine \\
32937 & Paroxetine \\
1040053 & Dextromethorphan/Quinidine \\
9068 & Quinidine \\
1007942 & Quinidine/Verapamil \\
1721552 & Florfenicol/Mometasone/terbinafine \\
37801 & Terbinafine \\
72625 & Duloxetine \\
36437 & Sertraline \\
\hline
\end{tabular}

Pharmacogenomics and Personalized Medicine

\section{Publish your work in this journal}

Pharmacogenomics and Personalized Medicine is an international, peerreviewed, open access journal characterizing the influence of genotype on pharmacology leading to the development of personalized treatment programs and individualized drug selection for improved safety, efficacy and sustainability. This journal is indexed on the American Chemical
Ingredient name

Bupropion/Naltrexone

Cinacalcet

Fluoxetine/olanzapine

Quinidine

Quinidine/Verapamil

Florfenicol/Mometasone/terbinafine

Terbinafine

Sertraline

Submit your manuscript here: https://www.dovepress.com/pharmacogenomics-and-personalized-medicine-journal 